\title{
Monadic dynamic algebras
}

\author{
S. Marques Pinto*, M. Teresa Oliveira-Martins" ${ }^{* *}$, and M. Céu Pinto*** \\ Departamento de Matemática, Universidade de Coimbra, 3001-454 Coimbra, Portugal
}

Received 29 July 2005, revised 11 November 2005, accepted 14 November 2005

Published online 1 March 2006

Key words Dynamic algebra, monadic algebra, monadic dynamic algebra, dynamic hemimorphism, dynamic relation, dual of a quantifier.

MSC (2000) 03B05, 03B70, 03G05, 06E15, 06E25

The main purpose of this work is to introduce the class of the monadic dynamic algebras (dynamic algebras with one quantifier). Similarly to a theorem of Kozen we establish that every separable monadic dynamic algebra is isomorphic to a monadic (possibly non-standard) Kripke structure. We also classify the simple (monadic) dynamic algebras. Moreover, in the dynamic duality theory, we analyze the conditions under which a hemimorphism of a dynamic algebra into itself defines a quantifier.

(c) 2006 WILEY-VCH Verlag GmbH \& Co. KGaA, Weinheim

Monadic Boolean algebras [1] and dynamic algebras [10, 11] are both recognizable as modal algebras [2, 3]. The fact that a monadic Boolean algebra is a very particular case of a dynamic algebra with only one action (the quantifier) led us to the study of a class of dynamic algebras, which we will call monadic dynamic algebras, where there exists an action behaving as a quantifier.

\section{Functional monadic Boolean algebras}

Functional monadic Boolean algebras were introduced by Halmos in [1]. The set $B^{X}$ of all functions from $X$ (a non-empty set) to $B$, where $\mathcal{B}=(B, \vee, \sim, 0)$ is a Boolean algebra, is itself a Boolean algebra $\mathcal{B}^{X}$ with respect to the pointwise operations, namely, if $p$ and $q$ are elements of $B^{X}$, then the supremum $p \vee q$ and the complement $\sim p$ are defined by

$$
(p \vee q)(x)=p(x) \vee q(x) \text { and } \quad(\sim p)(x)=\sim(p(x)),
$$

for every $x \in X$, and where the zero and the unit of $\mathcal{B}^{X}$ are, respectively, functions that are constantly equal to 0 and 1. Let us denote by $R(p)$ the range of the function $p$ of $B^{X}$. A Boolean subalgebra $\mathcal{A}$ of $\mathcal{B}^{X}$ such that

(i) for every $p$ in $A$ the supremum $\bigvee R(p)$ and the infimum $\bigwedge R(p)$ exist in $B$ and

(ii) the (constant) functions $\exists p$ and $\forall p$, defined by

$$
\exists p(x)=\bigvee R(p) \text { and } \forall p(x)=\bigwedge R(p),
$$

belong to $A$,

is called a functional monadic Boolean algebra or a B-valued functional monadic Boolean algebra with domain $X$. In this definition it is not necessary to impose that, for every $p$ in $A$, both $\exists p$ and $\forall p$ exist and belong to $A$, since $\forall p$ can be interpreted as $\sim(\exists(\sim p))$ and $\exists p$ as $\sim(\forall(\sim p))$. By the mutual duality of these operators we shall study $\exists$ alone. In particular, in the functional monadic Boolean algebra $2^{X}$, where $2=\{0,1\}$, if $p$ is a non-zero element of $2^{X}$, there exists an element $x_{0}$ in $X$ such that $p\left(x_{0}\right)=1$. Then $1 \in R(p)$ and $\exists p(x)=\bigvee R(p)=1$, for every $x \in X$. If $p=0$ in $2^{X}$, then $p(x)=0$, for every $x \in X$, and $\exists p(x)=\bigvee R(p)=0$. Therefore $2^{X}$ is a functional monadic Boolean algebra, where $\exists p=1$, for $p \neq 0$, and $\exists 0=0$.

* e-mail: sandra@mat.uc.pt

** Corresponding author: e-mail: meresa@mat.uc.pt

*** e-mail: pinto@mat.uc.pt 
If, for any set $Y$, we take $\mathcal{B}=\mathcal{P}(Y)$, the (complete) Boolean algebra of all the subsets of $Y$, then an arbitrary element $p$ in $\mathcal{B}^{X}$ is a function of $X$ in $\mathcal{P}(Y)$,

$$
p: X \longrightarrow \mathcal{P}(Y), \quad x \longmapsto P_{x}
$$

Immediately, for any $p \in \mathcal{B}^{X}, \exists p$ is defined (since $\mathcal{B}$ is complete) by

$$
\exists p: X \longrightarrow \mathcal{P}(Y), \quad x \longmapsto \exists p(x)=\bigvee\left\{p\left(x_{1}\right): x_{1} \in X\right\}=\bigcup_{x_{1} \in X} P_{x_{1}}
$$

and, similarly, $\forall p$ is defined by

$$
\forall p: X \longrightarrow \mathcal{P}(Y), \quad x \longmapsto \forall p(x)=\bigwedge\left\{p\left(x_{1}\right): x_{1} \in X\right\}=\bigcap_{x_{1} \in X} P_{x_{1}} .
$$

Consequently, if $y$ is a point in $Y$, the value $p(x)$ of a function $p$ in $\mathcal{B}^{X}$ corresponds in a natural way to the proposition " $y$ belongs to $p(x)$ ". Since supremum is a set-theoretic union, it follows that each value of $\exists p$ corresponds to "there exists $x$ such that $y$ belongs to $p(x)$ " and, dually, that each value of $\forall p$ corresponds to "for every $x, y$ belongs to $p(x)$ ". For this reason, Halmos calls this operator $\exists$ (on a functional monadic Boolean algebra) a functional existential quantifier, and the operator $\forall$ a functional universal quantifier.

The functional existential quantifier $\exists$ on a functional monadic Boolean algebra $\mathcal{A}$ is normalized, increasing and quasi-multiplicative, i. e. satisfies

$$
\begin{aligned}
& (\exists)_{1} \exists 0=0, \\
& (\exists)_{2} p \leq \exists p, \\
& (\exists)_{3} \exists(p \wedge \exists q)=\exists p \wedge \exists q,
\end{aligned}
$$

whenever $p$ and $q$ are in $A$.

\section{Monadic Boolean algebras}

A general concept of quantification is obtained by abstraction from the functional case. A quantifier (an existential quantifier) on a Boolean algebra $\mathcal{B}$ is a mapping $\exists$ of the Boolean algebra $\mathcal{B}$ into itself satisfying $(\exists)_{1},(\exists)_{2}$ and $(\exists)_{3}$, for every $p, q$ in $B$.

The concept of a universal quantifier is defined by dualization, via the equation $\forall p=\sim(\exists(\sim p))$. Since we will only refer to universal quantifiers briefly, the adjective "existential" will usually be omitted.

It is worthwhile to look at some other examples of quantifiers (other than the functional one), namely the simple quantifier $\exists_{s}$, and the discrete quantifier $\exists_{d}$. The identity mapping of a Boolean algebra into itself is a quantifier, the discrete quantifier. The mapping defined by $\exists_{s} 0=0$ and $\exists_{s} p=1$, for every $p \neq 0$, is a quantifier, called the simple quantifier.

A quantifier $\exists$ on a Boolean algebra $\mathcal{B}$ satisfies the following algebraic properties (cf. [1]) for every $p, q$ in $B$ :

$\left(P_{1}\right) \exists 1=1$;

$\left(P_{2}\right) \exists \exists=\exists$;

$\left(P_{3}\right) p \in \exists(B)$ if and only if $\exists p=p$, where $\exists(B)$ is the range of $\exists$;

$\left(P_{4}\right)$ if $p \leq \exists q$, then $\exists p \leq \exists q$;

$\left(P_{5}\right)$ if $p \leq q$, then $\exists p \leq \exists q$ (the quantifier is monotone);

$\left(P_{6}\right) \exists(\sim(\exists p))=\sim(\exists p)$;

$\left(P_{7}\right)$ the range $\exists(B)$ of a quantifier $\exists$ is a Boolean subalgebra of $\mathcal{B}$;

$\left(P_{8}\right) \exists(p \vee q)=\exists p \vee \exists q$.

A monadic Boolean algebra is a Boolean algebra $\mathcal{B}$ together with a quantifier $\exists$ on $\mathcal{B}$. The elementary algebraic theory of monadic Boolean algebras is defined similarly to that of every other algebraic system. 


\section{Dynamic algebras}

Dynamic algebras were introduced by Kozen [6] and Pratt [10] to provide models for propositional dynamic logic (PDL). Following Pratt a dynamic algebra is a two-sorted algebra $(\mathcal{B}, \mathcal{R},\langle\rangle)$ verifying a certain set of equations. The Boolean universe $\mathcal{B}=(B, \vee, \sim, 0)$ is a Boolean algebra (an algebra with a binary operation $\vee$, one unary operation $\sim$ and a nullary operation 0 , satisfying the usual set of axioms); the regular universe $\mathcal{R}=\left(R, \cup, ;,^{*}\right)$ (or universe of actions or universe of programs) is an algebra with two binary operations, $\cup$ and ; , and one unary operation *. The heterogeneous operator (diamond) \langle\rangle$: R \times B \longrightarrow B$ relates both algebras.

Definition 3.1 A dynamic algebra $\mathcal{D}=(\mathcal{B}, \mathcal{R},\langle\rangle)$, where $\mathcal{B}=(B, \vee, \sim, 0), \mathcal{R}=\left(R, \cup, ;{ }^{*}\right)$, is an algebra satisfying, for $p, q \in B$ and $a, b \in R$,

(1) $\mathcal{B}$ is a Boolean algebra;

(2) $\langle a, 0\rangle=0,\langle a, p \vee q\rangle=\langle a, p\rangle \vee\langle a, q\rangle$;

(3) $\langle a \cup b, p\rangle=\langle a, p\rangle \vee\langle b, p\rangle$;

(4) $\langle a ; b, p\rangle=\langle a,\langle b, p\rangle\rangle$;

(5) $p \vee\left\langle a ; a^{*}, p\right\rangle \leq\left\langle a^{*}, p\right\rangle$;

(6) $\left\langle a^{*}, p\right\rangle \leq p \vee\left\langle a^{*}, \sim p \wedge\langle a, p\rangle\right\rangle$.

\section{Remark 3.2}

1. For $p$ and $q$ elements of the Boolean algebra $\mathcal{B}$, we used $(p \leq q)$ as an abbreviation of $(p \vee q=q)$.

2. Pratt [10] showed that conditions (5) and (6) can be replaced by $\left\langle a^{*}, p\right\rangle=\min a ! p$, where

$$
a ! p=\{q \in B: p \vee a q \leq q\} .
$$

Notation 3.3 For $a, b \in R$ and $p \in B$ we write $a p$ instead of $\langle a, p\rangle, a b$ instead of $a$; $b$, and we write $a \leq b$ whenever $a p \leq b p$ for every $p \in B$.

Dynamic algebras, as defined by Kozen [7], have one more operation, called reversion. A dynamic algebra with reversion is a dynamic algebra $\mathcal{D}=(\mathcal{B}, \mathcal{R},\langle\rangle)$ equipped with a unary operation ${ }^{-}$on $\mathcal{R}$ such that $a^{--}=a$, $(a \cup b)^{-}=a^{-} \cup b^{-},(a b)^{-}=b^{-} a^{-}$and

$$
p \leq \sim a\left(\sim a^{-} p\right), \quad \text { for } a, b \in R, p \in B .
$$

A dynamic algebra $(\mathcal{B}, \mathcal{R},\langle\rangle)$ is *-continuous if, for every $a \in R$ and $p \in B, a^{*} p=\vee\left\{a^{i} p: i \geq 0\right\}$.

Kripke structures, traditional models for PDL, were presented in [10] as examples of dynamic algebras. They are defined as follows. The full Kripke structure on a given non-empty set $S$ is a triple $(S, \mathcal{P}(S), \mathcal{P}(S \times S))$, where $\mathcal{P}(S)$ is the Boolean algebra of all subsets of $S$ (with the usual set theoretical operations) and $\mathcal{P}(S \times S)$ is the set of all binary relations on $S$ endowed with the operations $;, \cup$, and ${ }^{*}$ as, respectively, the composition, the union and the reflexive-transitive closure of binary relations, and where the diamond operation $\langle a, p\rangle$ (for $a \in \mathcal{P}(S \times S)$ and $p \in \mathcal{P}(S))$ is the pre-image of $p$ under $a$, i. e.

$$
\langle a, p\rangle=\left\{s \in S:\left(s, s^{\prime}\right) \in a, \text { for some } s^{\prime} \in p\right\} .
$$

Kripke structures (or standard Kripke structures) are defined as the dynamic subalgebras of full Kripke structures. A dynamic algebra defined as a Kripke structure for all operations but, possibly, the operation * (defined as any unary operation of binary relations satisfying the axioms (5) and (6)) is called non-standard Kripke structure. Every standard Kripke structure is a ${ }^{*}$-continuous dynamic algebra. In a Kripke structure the reversion of a binary relation $a$ is interpreted as its inverse relation, $a^{-}=\{(s, t):(t, s) \in a\}$.

Let $\mathcal{D}$ be a dynamic algebra. Two actions $a, b$ are inseparable, and we denote that fact by $a \cong b$, if $a p=b p$, for every proposition $p$ in $B$. The pair $(=, \cong$ ) defines a congruence relation (cf. Definition 3.8 below) in the dynamic algebra $\mathcal{D}$. A dynamic algebra is separable if the inseparable relation is the identity relation, i. e. for any actions $a$ and $b$ in $R$, if $a p=b p$, for any proposition $p \in B$, then $a=b$.

The mixed operator diamond \langle\rangle$: R \times B \longrightarrow B$ can be seen as

$$
\langle\rangle: R \longrightarrow F(B ; B), \quad a \longmapsto(p \longmapsto\langle a\rangle p=\langle a, p\rangle),
$$

where $F(B ; B)=\{f: f: B \longrightarrow B$ is a mapping $\}$. However, unless $\mathcal{D}$ is separable, \langle\rangle does not define a representation of $R$ since \langle\rangle is not one-to-one. We may have equal mappings $\langle a\rangle$ and $\langle b\rangle$ in $F(B ; B)$ with $a \neq b$ and, 
consequently, for $\mathcal{D}$ an arbitrary dynamic algebra, $\{\langle a\rangle: a \in R\}$ is a family of mappings in $B$ indexed by $R$ (where the extensionality axiom is not satisfied). In a separable dynamic algebra, $R$ may be identified with a set of mappings in $B$, that we will use whenever appropriate.

The relation $\leq$ in $R, a \leq b$ whenever $a p \leq b p$, for every $p \in B$, is reflexive and transitive, but it is not antisymmetric (since the conditions $a p \leq b p$ and $b p \leq a p$ give rise to $a p=b p$, for every $p \in B$ and not necessarily to $a=b$ ) and, therefore, defines a quasi-order in $R$. Clearly, in separable dynamic algebras $\leq$ defines a partial order since the antisymmetry is satisfied.

Example 3.4 Every monadic Boolean algebra defines a dynamic algebra. In fact, let $\mathcal{B}$ be a Boolean algebra with a quantifier $\exists_{1}, \mathcal{R}=\left(\left\{\exists_{1}\right\}, \cup, ;{ }^{*}\right)$, where $\cup$, and ${ }^{*}$ are defined trivially, and \langle\rangle$:\left\{\exists_{1}\right\} \times B \longrightarrow B$ is the application defined by $\left\langle\exists_{1}, p\right\rangle:=\exists_{1}(p)$, for every $p \in B$. Since $\exists_{1} 0=0, \exists_{1}(p \vee q)=\exists_{1} p \vee \exists_{1} q$, for every $p, q \in B, \exists_{1} \exists_{1}=\exists_{1}$ and $\exists_{1}^{*}=\exists_{1},(\mathcal{B}, \mathcal{R},\langle\rangle)$ is a dynamic algebra.

Conversely, we may ask if every dynamic algebra with a unique element in the regular part defines a monadic Boolean algebra. The next example shows that the answer to this question is negative.

Example 3.5 Let $\mathcal{D}=(\mathcal{B}, \mathcal{R},\langle\rangle)$ be the Kripke structure on $S=\{1,2,3\}$, where

$$
B=\{\emptyset,\{3\},\{1,2\},\{1,2,3\}\}
$$

and $R=\{a\}$ with $a=\{(1,1),(1,2),(2,2),(3,2),(3,3)\}$. Notice that $a=a \cup a=a a=a^{*}$ and $a p \in B$, for every $p \in B$. Therefore $\mathcal{D}$ is a dynamic algebra. The regular element $a$ does not define a quantifier on $\mathcal{B}$. For, let $A: B \longrightarrow B$ be the operator defined by $a$, i. e. $A(p):=\langle a\rangle p$, for every $p \in B$. Since $\sim A\{3\}=\{1,2\}$ and $A(\sim A\{3\})=\{1,2,3\}$, the property of quantifiers, $\left(P_{6}\right)$, is not satisfied.

Although, for dynamic algebras with reversion the answer is positive, as we will see in the next proposition.

Proposition 3.6 Every dynamic algebra with reversion $\mathcal{D}=(\mathcal{B},\{a\},\langle\rangle)$ defines a monadic Boolean algebra.

Proof. Let $\mathcal{D}=(\mathcal{B},\{a\},\langle\rangle)$ be a dynamic algebra with reversion. We prove that the application $A$ on $\mathcal{B}$ defined by $A(p):=a p$, for every $p \in B$, is a quantifier on $\mathcal{B}$. For that, it is enough to prove that $A$ is a closure operator and that $A(\sim(A p))=\sim(A p)$, for any $p \in B$ (cf. [1, Theorem 3]).

(i) $A 0=0, A A(p)=A(p)$ and $A(p \vee q)=A p \vee A q$, for every $p, q \in B$, is a consequence, respectively, of the fact that $a$ is normal $(a 0=0)$, idempotent and additive.

(ii) Since $a=a^{*}$ and $p \leq a^{*} p$, for every $p \in B$, we have $p \leq a p$ and so, $p \leq A p$, for every $p \in B$. Therefore $A$ is a closure operator on $B$.

(iii) By (ii) we have $\sim(A p) \leq A(\sim(A p))$, for every $p \in B$. By (1) we know that, for every $p \in B$, we have $p \leq \sim a\left(\sim a^{-} p\right)$. Then $a\left(\sim a^{-} p\right) \leq \sim p$, and since $a=a^{-}$, we have $a(\sim a p) \leq \sim p$. Changing $p$ to $a p$, we get $a(\sim a(a p)) \leq \sim(a p)$ and, since $a=a a, a(\sim(a p)) \leq \sim(a p)$. Therefore $A(\sim(A p)) \leq \sim(A p)$, for every $p \in B$.

The elementary algebraic theory of dynamic algebras is defined similarly to that of every other algebraic system $[13,14]$. We will specify some concepts for later use.

Definition 3.7 Let $\mathcal{D}=(\mathcal{B}, \mathcal{R},\langle\rangle)$ and $\mathcal{D}^{\prime}=\left(\mathcal{B}^{\prime}, \mathcal{R}^{\prime},\langle\rangle^{\prime}\right)$ be dynamic algebras, where $\mathcal{B}=(B, \vee, \sim, 0)$, $\mathcal{R}=\left(R, \cup, ;{ }^{*}\right), \mathcal{B}^{\prime}=\left(B^{\prime}, \vee^{\prime}, \sim^{\prime}, 0^{\prime}\right)$, and $\mathcal{R}^{\prime}=\left(R^{\prime}, \cup^{\prime}, ;^{\prime},{ }^{* \prime}\right)$, and let $h_{1}: B \longrightarrow B^{\prime}$ and $h_{2}: R \longrightarrow R^{\prime}$ be mappings. The pair $h=\left(h_{1}, h_{2}\right)$ is a dynamic homomorphism between the dynamic algebras $\mathcal{D}$ and $\mathcal{D}^{\prime}$ if $h_{1}$ and $h_{2}$ preserve the mentioned one-sorted operations and $h$ preserves the mixed operation, i.e. for $p \in B$ and $a, b \in R$ we have

1. $h_{1}$ is a Boolean homomorphism;

2. $h_{2}(a \cup b)=h_{2}(a) \cup^{\prime} h_{2}(b)$;

3. $h_{2}(a ; b)=h_{2}(a) ;^{\prime} h_{2}(b)$;

4. $h_{2}\left(a^{*}\right)=\left(h_{2}(a)\right)^{* \prime}$;

5. $h_{1}(\langle a\rangle p)=\left\langle h_{2}(a)\right\rangle^{\prime} h_{1}(p)$. 
Definition 3.8 Let $\mathcal{D}=(\mathcal{B}, \mathcal{R},\langle\rangle)$ be a dynamic algebra. The pair $\theta=\left(\theta_{1}, \theta_{2}\right)$ is a (dynamic) congruence relation in $\mathcal{D}$ if $\theta_{1}$ is a congruence relation in $\mathcal{B}, \theta_{2}$ is a congruence relation in $\mathcal{R}$ and, moreover, if $p \theta_{1} q$ and $a \theta_{2} b$, then $a p \theta_{1} b q$, for every $p, q \in B$ and $a, b \in R$.

As usual, we define $\mathcal{B} / \theta_{1}=\left\{[p]_{\theta_{1}}: p \in B\right\}$ and $\mathcal{R} / \theta_{2}=\left\{[a]_{\theta_{2}}: a \in R\right\}$. The structure

$$
\mathcal{D} / \theta=\left(\mathcal{B} / \theta_{1}, \mathcal{R} / \theta_{2},\langle\rangle\right)
$$

is a dynamic algebra (the quotient dynamic algebra) with operations defined by

$$
\begin{array}{lll}
{[p]_{\theta_{1}} \vee[q]_{\theta_{1}}:=[p \vee q]_{\theta_{1}},} & {[a]_{\theta_{2}} \cup[b]_{\theta_{2}}:=[a \cup b]_{\theta_{2}},} & \sim[p]_{\theta_{1}}:=[\sim p]_{\theta_{1}} \\
{[a]_{\theta_{2}} ;[b]_{\theta_{2}}:=[a ; b]_{\theta_{2}},} & {[a]_{\theta_{2}}[p]_{\theta_{1}}:=[a p]_{\theta_{1}},} & {[a]_{\theta_{2}}^{*}:=\left[a^{*}\right]_{\theta_{2}} .}
\end{array}
$$

$\left(\mathcal{B} / \theta_{1}, \mathcal{R} / \theta_{2},\langle\rangle\right)$ is the homomorphic image of $(\mathcal{B}, \mathcal{R},\langle\rangle)$ under the natural homomorphism $\nu=\left(\nu_{1}, \nu_{2}\right)$ defined by $\nu_{1}: p \longmapsto[p]_{\theta_{1}}, \nu_{2}: a \longmapsto[a]_{\theta_{2}}$ for $p \in B, a \in R$.

Also, associated with each homomorphism, there exists a natural congruence. If $h: \mathcal{D} \longrightarrow \mathcal{D}^{\prime}$ is a dynamic algebra homomorphism, the kernel relation $\operatorname{ker}_{h}=\left(K_{1}, K_{2}\right)$ given by

$$
p K_{1} q \text { if and only if } h_{1}(p)=h_{1}(q), \quad a K_{2} b \text { if and only if } h_{2}(a)=h_{2}(b)
$$

for every $p, q \in B$ and $a, b \in R$, defines a congruence in $\mathcal{D}$.

The homomorphism theorem enables us to assert that every homomorphic image of a dynamic algebra $\mathcal{D}$ is, up to an isomorphism, a quotient algebra [14].

\section{Quantified propositional dynamic logic}

Propositional dynamic logic PDL is being used in the literature to describe different logic systems [4, 5, 8]. Depending on the problem we have to solve, we use different connectors, - (reversion) [6, 7] and ? (test) [10, 12]. Similarly, in this work, we devote ourselves to the study of a new connector, the existential quantifier, $\exists$. We use quantified propositional dynamic logic, QPDL, to denote the version of PDL with quantifier(s).

\subsection{Syntax}

The expressions of QPDL are of two sorts: propositional sort (propositions or formulae) and regular sort (actions or programs). All these expressions are built by recursion from a numerable set of propositional variables (atomic propositions), a numerable set of regular variables (atomic programs), constants 0, 1 of propositional sort, constant $\exists_{0}$ of regular sort, propositional connectors $\vee, \sim, \exists_{1}$, program connectors $\cup$ (non-deterministic choice), ; (composition), * (iteration), $\exists_{2}$, a mixed connector \langle\rangle (diamond) and (, ) (parenthesis).

The formation of propositions and programs is subject to the following rules:

1. Constants 0,1 are propositions and constant $\exists_{0}$ is a program.

2. Each atomic proposition is a proposition and each atomic program is a program.

3. If $p$ and $q$ are propositions, then $(p \vee q),(\sim p)$ and $\left(\exists_{1} p\right)$ are propositions.

4. If $a$ and $b$ are programs, then $(a \cup b),(a ; b),\left(a^{*}\right)$ and $\left(\exists_{2} a\right)$ are programs.

5. If $p$ is a proposition and $a$ is a program, then $\langle a\rangle p$ is a proposition.

\subsection{Semantics}

The semantic structures of QPDL are pairs $\mathcal{S}=(\mathcal{P}(S), \mathcal{P}(S \times S))$ associated with interpretation maps $\mathcal{I}$, where $S$ is a non-empty finite set, called the set of states, $\mathcal{P}(S)$ is the family of all the subsets of $S$ and $\mathcal{P}(S \times S)$ is the family of all the binary relations defined on $S$. Therefore, QPDL expressions are interpreted in semantic structures corresponding each atomic proposition $p$ to a subset $\mathcal{I}_{p}$ of $S$ and each atomic program $a$ to 
a binary relation $\mathcal{I}_{a} \subseteq S \times S$. The interpretation of non-atomic expressions results from the interpretation of atomic expressions by using induction on their syntactic structure. More precisely, an interpretation $\mathcal{I}$ of QPDL expressions in a semantic structure $\mathcal{S}=(\mathcal{P}(S), \mathcal{P}(S \times S))$ is defined by:

1. $\mathcal{I}_{0}=\emptyset, \mathcal{I}_{1}=S, \mathcal{I}_{\exists_{0}}$ is an equivalence relation on $S$.

2. Each atomic proposition $p$ is interpreted as a subset $\mathcal{I}_{p}$ of $S$.

3. Each atomic program $a$ is interpreted as a binary relation $\mathcal{I}_{a}$ on $S$.

4. $\mathcal{I}_{\langle a\rangle p}=\left\{s \in S:\left(s, s_{1}\right) \in \mathcal{I}_{a}\right.$, for some $\left.s_{1} \in \mathcal{I}_{p}\right\}$ (is the pre-image of $\mathcal{I}_{p}$ under $\mathcal{I}_{a}$ ).

5. $\mathcal{I}_{p \vee q}=\mathcal{I}_{p} \cup \mathcal{I}_{q}, \mathcal{I}_{\sim p}=\mathcal{I}_{p}^{\mathrm{c}}, \mathcal{I}_{\exists_{1} p}=\mathcal{I}_{\left\langle\exists_{0}\right\rangle p}$.

6. $\mathcal{I}_{a \cup b}=\mathcal{I}_{a} \cup \mathcal{I}_{b}$,

$$
\mathcal{I}_{a ; b}=\mathcal{I}_{a} \circ \mathcal{I}_{b}=\left\{\left(s_{1}, s_{2}\right):\left(s_{1}, s_{3}\right) \in \mathcal{I}_{a} \text { and }\left(s_{3}, s_{2}\right) \in \mathcal{I}_{b} \text {, for some } s_{3} \in S\right\},
$$

$\mathcal{I}_{a^{*}}=\bigcup_{n \in \mathbb{N}_{0}} \mathcal{I}_{a}^{n}$ with $\mathcal{I}_{a}^{0}=\Delta_{S}=\{(s, s): s \in S\}$ and $\mathcal{I}_{a}^{n}=\mathcal{I}_{a} \circ \mathcal{I}_{a}^{n-1}, n \in \mathbb{N}, \mathcal{I}_{\exists_{2} a}=\mathcal{I}_{\exists_{0} ; a}$.

Remark 4.1 The symbols $\cup,{ }^{c}$ used on the right-hand side of the identities denote the set operations of union and complementation, respectively, and the symbol $\circ$ denotes composition of binary relations.

We may see the set $S$ as the set of possible states of a computer, the propositions as subsets of states and the actions as computer programs. Therefore, $s \in \mathcal{I}_{p}$ means that "the state $s$ satisfies proposition $p$ ", $\left(s_{1}, s_{2}\right) \in \mathcal{I}_{a}$ means that "the program $a$ may begin at state $s_{1}$ and finish at $s_{2}$ ". The propositional operations are interpreted in a natural form. To have $s \in \mathcal{I}_{p \vee q}$ means that " $s$ satisfies proposition $p$ or proposition $q$ ", $s \in \mathcal{I}_{\sim p}$ means that "the state $s$ does not satisfy proposition $p$ " and $\exists_{1} p$ means that "there exists a state satisfying $p$, where is possible to execute $\exists_{0}$ ". The program $a \cup b$ means "choose $a$ or $b$ non-deterministically and execute it", $a ; b$ means "execute program $a$ and then $b$ ", $a^{*}$ means "choose $n \in \mathbb{N}_{0}$, and execute $n$ times program $a$ " and $\exists_{2} a$ means "to execute program $\exists_{0}$ and then $a$ ". Finally, $s \in \mathcal{I}_{\langle a\rangle p}$ means that "it is possible to execute $a$ beginning at $s$ and finish at a state satisfying proposition $p "$.

\subsection{Monadic Kripke structures}

We are going to use the QPDL to introduce a structure called monadic Kripke structure.

Proposition 4.2 If $S$ is a non-empty set and $\exists_{0} \subseteq S \times S$ is an equivalence relation on $S$, then $\exists_{1}$ defined by

$$
\exists_{1} P:=\exists_{0}^{-1}(P)=\left\{s_{1} \in S:\left(s_{1}, s_{2}\right) \in \exists_{0}, \text { for some } s_{2} \in P\right\}
$$

is a quantifier on any Boolean subalgebra, $\mathcal{B}$, of $\mathcal{P}(S)$ whenever it satisfies $\exists_{1} B \subseteq B$.

Proof. To prove that $\exists_{1}$ is a quantifier on $\mathcal{B}$ it is enough (cf. [1, Theorem 3]) to verify that $\exists_{1}$ is a closure operator and that $\exists_{1}\left(\exists_{1} P\right)^{\mathrm{c}}=\left(\exists_{1} P\right)^{\mathrm{c}}$, for any $P \in B$.

(i) The condition $\exists_{1} \emptyset=\emptyset$ is verified trivially.

(ii) $P \subseteq \exists_{1} P$, for every $P \in \mathcal{B}$, since $\exists_{0}$ is reflexive on $S$.

(iii) $\exists_{1}\left(\exists_{1} P\right)=\exists_{1} P$, for every $P \in \mathcal{B}$. In fact, by (ii) we have $\exists_{1} P \subseteq \exists_{1}\left(\exists_{1} P\right)$. Now, let $s_{1} \in \exists_{1}\left(\exists_{1} P\right)$. Then $\left(s_{1}, s_{2}\right) \in \exists_{0}$, for some $s_{2} \in \exists_{1} P$, and so $\left(s_{2}, s_{3}\right) \in \exists_{0}$, for some $s_{3} \in P$. Since $\exists_{0}$ is transitive, then $\left(s_{1}, s_{3}\right) \in \exists_{0}$. Hence, we have $s_{1} \in \exists_{1} P$.

(iv) $\exists_{1}(P \cup Q)=\exists_{1} P \cup \exists_{1} Q$ since $\exists_{1} U$ is defined as the pre-image of $U$ under the (equivalent) relation $\exists_{0}$, for any subset $U$ of $S$.

Using (i), (ii), (iii) and (iv) we may conclude that $\exists_{1}$ is a closure operator. It remains to prove

(v) $\exists_{1}\left(\exists_{1} P\right)^{\mathrm{c}}=\left(\exists_{1} P\right)^{\mathrm{c}}$. By (ii) we have $\left(\exists_{1} P\right)^{\mathrm{c}} \subseteq \exists_{1}\left(\exists_{1} P\right)^{\mathrm{c}}$. Let $s \in \exists_{1}\left(\exists_{1} P\right)^{\mathrm{c}}$. Then $\left(s, s_{2}\right) \in \exists_{0}$, for some $s_{2} \in\left(\exists_{1} P\right)^{\mathrm{c}}$. So, if $\left(s_{2}, z\right) \in \exists_{0}$, then $z \notin P$. Consider $\left(s, z_{1}\right) \in \exists_{0}$. Since $\left(s, s_{2}\right) \in \exists_{0}$, then $\left(s_{2}, s\right) \in \exists_{0}$. But since $\exists_{0}$ is transitive we have $\left(s_{2}, z_{1}\right) \in \exists_{0}$ and hence $z_{1} \notin P$. We conclude that if $\left(s, z_{1}\right) \in \exists_{0}$, then $z_{1} \notin P$, i. e. $s \notin \exists_{1} P$, and thus, $s \in\left(\exists_{1} P\right)^{\mathrm{c}}$.

Let $S$ be a non-empty set (of states) and $\mathcal{I}$ an interpretation of QPDL in $S$. Then the set of all $\mathcal{I}_{p}$, with $p$ running over all propositions of QPDL, is a monadic Boolean algebra (with quantifier corresponding to $\mathcal{I}_{\exists_{0}}$, by Proposition 4.2) under union and complementation of subsets of $S$. We denote such an algebra by $B(\mathcal{I})$. 
The set of all the $\mathcal{I}_{a}$, with $a$ running over all the programs in QPDL, is a Kleene algebra (i.e. is a set of binary relations closed under union, composition and reflexive-transitive closure of binary relations) containing an equivalence relation of domain $S$. We denote it by $R(\mathcal{I})$. Moreover, for each $\mathcal{I}_{p} \in B(\mathcal{I})$ and $\mathcal{I}_{a} \in R(\mathcal{I})$,

$$
\left\langle\mathcal{I}_{a}\right\rangle \mathcal{I}_{p}=\mathcal{I}_{\langle a\rangle p}=\left\{s \in S:\left(s, s_{1}\right) \in \mathcal{I}_{a}, \text { for some } s_{1} \in \mathcal{I}_{p}\right\}
$$

is in $B(\mathcal{I})$, i. e. $B(\mathcal{I})$ is closed under images of elements of $B(\mathcal{I})$ by relations in $R(\mathcal{I})$.

We define a monadic Kripke structure $(S, \mathcal{B}, \mathcal{R}, \exists)$ as a Kripke structure $(S, \mathcal{B}, \mathcal{R})$ with an operator

$$
\exists=\left(\exists_{0}, \exists_{1}, \exists_{2}\right),
$$

where $\exists_{0}$ is an equivalence relation on $S$ belonging to $R, \exists_{1}$ is an application on $\mathcal{B}$ defined, for $P \in B$, by

$$
\exists_{1} P:=\left\langle\exists_{0}\right\rangle P=\left\{s_{1} \in S:\left(s_{1}, s_{2}\right) \in \exists_{0}, \text { for some } s_{2} \in P\right\},
$$

and $\exists_{2}$ is an application on $R$ defined by $\exists_{2} a=\exists_{0} ; a$, for every $a \in R$.

Immediately, every interpretation of QPDL defines a monadic Kripke structure.

\section{Monadic dynamic algebra}

By abstracting the notion of a monadic Kripke structure, we are led to the definition of a monadic dynamic algebra.

Definition 5.1 A monadic dynamic algebra is a quadruple $(\mathcal{B}, \mathcal{R},\langle\rangle, \exists)$, where $(\mathcal{B}, \mathcal{R},\langle\rangle)$ is a dynamic algebra and $\exists$ is a triple $\left(\exists_{0}, \exists_{1}, \exists_{2}\right)$ such that

1. $\exists_{0} \in R$;

2. $\exists_{1}: B \longrightarrow B$, where $\exists_{1}(p):=\left\langle\exists_{0}\right\rangle p$, for every $p \in B$, is a quantifier on $\mathcal{B}$;

3. $\exists_{2}: R \longrightarrow R$ is defined by $\exists_{2}(a):=\exists_{0} ; a$, for every $a \in R$.

The operator $\exists$ is called a dynamic quantifier.

In the following, given a monadic dynamic algebra $\mathcal{D}_{M}=(\mathcal{B}, \mathcal{R},\langle\rangle, \exists)$ we denote by $\mathcal{D}=(\mathcal{B}, \mathcal{R},\langle\rangle)$ its reduct which is a dynamic algebra.

A monadic dynamic algebra $\mathcal{D}_{M}=(\mathcal{B}, \mathcal{R},\langle\rangle, \exists)$ is called separable if it is separable as dynamic algebra, i. e. if $\mathcal{D}$ is separable.

Remark 5.2 In Definition 5.1, if the monadic dynamic algebra $(\mathcal{B}, \mathcal{R},\langle\rangle, \exists)$ is separable, then there exists only the element $\exists_{0}$ in $R$ such that $\exists_{1}(p)=\left\langle\exists_{0}\right\rangle p$, for every $p \in B$. Thus, for these algebras we will write $\exists_{1}$ to represent either the regular element $\exists_{0}$ or the Boolean operator $\exists_{1}$.

Hence, we can say that a separable monadic dynamic algebra is a quadruple $(\mathcal{B}, \mathcal{R},\langle\rangle, \exists)$, where $(\mathcal{B}, \mathcal{R},\langle\rangle)$ is a dynamic algebra and $\exists$ is a pair $\left(\exists_{1}, \exists_{2}\right)$ such that

1. $\exists_{1} \in R$;

2. $\exists_{1}: B \longrightarrow B$, where $\exists_{1}(p):=\left\langle\exists_{1}\right\rangle p$, for every $p \in B$, is a quantifier on $\mathcal{B}$;

3. $\exists_{2}: R \longrightarrow R$ is defined by $\exists_{2}(a):=\exists_{1} ; a$, for every $a \in R$.

Example 5.3 Let $X$ be a non empty set and $\mathcal{B}=(B, \vee, \sim, 0)$ a complete Boolean algebra. Then the set of all the functions of $X$ into $B, \mathcal{B}^{X}$, is itself a complete Boolean algebra. The functional quantifier $\exists_{1}$ enables us to qualify $\mathcal{B}=\left(B, \vee, \sim, 0, \exists_{1}\right)$ as a (functional) monadic Boolean algebra (cf. Section 1$)$. For $\mathcal{R}=\left(R, \cup, ;,{ }^{*}\right)$, where $R$ is the set of all the normal and completely additive functions on $\mathcal{B}^{X}, \cup, ;{ }^{*}$ are, respectively, the pointwise disjunction, composition and reflexive-transitive closure of elements of $R$, and \langle\rangle$: R \times \mathcal{B}^{X} \longrightarrow \mathcal{B}^{X}$ is the map defined by

$$
\langle a, p\rangle:=a(p), \quad \text { for every } a \in R, p \in B,
$$

we know that $\left(\mathcal{B}^{X}, \mathcal{R},\langle\rangle\right)$ is a separable dynamic algebra (the completely full dynamic algebra on $\mathcal{B}^{X}$ ), see [10]. Therefore $\left(\mathcal{B}^{X}, R,\langle\rangle, \exists\right)$ with $\exists=\left(\exists_{1}, \exists_{2}\right)$ given by

$$
\exists_{1} p(x)=\bigvee\left\{p\left(x_{1}\right): x_{1} \in X\right\} \quad \text { and } \quad \exists_{2}(a)=\exists_{1} ; a, \quad \text { for } a \in R, p \in B,
$$

is a monadic dynamic algebra. 
Example 5.4 By Proposition 3.6 we can easily see that every dynamic algebra $\mathcal{D}_{M}=(\mathcal{B},\{a\},\langle\rangle)$ with reversion defines a monadic dynamic algebra $\mathcal{D}=(\mathcal{B},\{a\},\langle\rangle, \exists)$ with $\exists=\left(\exists_{1}, \exists_{2}\right)$ given by $\exists_{1}(p):=a p$, for every $p \in B$, and $\exists_{2}(a):=a$.

We can derive a list of elementary consequences of the definition of monadic dynamic algebra.

Proposition 5.5 In any monadic dynamic algebra $\mathcal{D}_{M}=(\mathcal{B}, \mathcal{R},\langle\rangle, \exists)$, the following assertions are satisfied:

1. $\exists_{0}^{*} p=\exists_{0} p$, for every $p \in B$, i.e. $\exists_{0}$ is an asterate.

2. $\left(\exists_{2}\left(\exists_{0}\right)\right) p=\exists_{0}$, for every $p \in B$.

3. $a \leq \exists_{2}(a)$, for every $a \in R$.

4. If $\mathcal{D}$ is separable and $0_{R} \in R$, then $\exists_{2}\left(0_{R}\right)=0_{R}$.

5. If $\mathcal{D}$ is separable and $1_{R} \in R$, then $\exists_{2}\left(1_{R}\right)=1_{R}$.

6. $\left(\exists_{2}\left(\exists_{2}(a)\right)\right) p=\left(\exists_{2}(a)\right) p$, for every $p \in B$.

7. If $\mathcal{D}$ is separable, $a \in \exists_{2}(R)$ if and only if $a=\exists_{2}(a)$.

8. If $a \leq \exists_{2}(b)$, then $\exists_{2}(a) \leq \exists_{2}(b)$.

9. If $a \leq b$, then $\exists_{2}(a) \leq \exists_{2}(b)$.

10. $\left(\exists_{2}(a \cup b)\right) p=\left(\exists_{2}(a) \cup \exists_{2}(b)\right) p$, for every $p \in B$.

11. $\left(\exists_{2}\left(a ; \exists_{2}(b)\right)\right) p=\left(\left(\exists_{2}(a)\right) ;\left(\exists_{2}(b)\right)\right) p$, for every $p \in B$.

(Here $0_{R}$ and $1_{R}$ denote constant maps of $B$ into $B$ equal to, respectively, 0 and 1.$)$

Remark 5.6 Since $a^{*} 0=0, p \leq a^{*} p$ and $a^{*} a^{*} p=a^{*} p$, for every $p \in B$ and $a \in R$ in a dynamic algebra $\mathcal{D}=(B, R,\langle\rangle)$, one could question if, for each $a \in R$, $a^{*}$ defines a quantifier on $\mathcal{B}$, i. e. if $\exists_{1}: B \longrightarrow B$ given by $\exists_{1} p=a^{*} p$ is a quantifier on $B$. The answer to this question is negative. In fact, let $B=\{0,1, p, \sim p\}$ and

$$
R=\{a: a: B \longrightarrow B \text { is a map, } a(0)=0 \text { and } a \text { is finitely additive }\},
$$

where $a(q)=q$ for every $q \neq \sim p$ and $a(\sim p)=1$. We have

$$
a^{*} p=\min \{q \in B: p \vee a q \leq q\}=\min \{p, 1\}=p .
$$

Therefore $a^{*}\left(\sim a^{*} p\right)=a^{*}(\sim p)=\min \{q \in B: \sim p \vee a q \leq q\}=1$. But $\sim a^{*} p=\sim p$. So $a^{*}\left(\sim a^{*} p\right) \neq \sim a^{*} p$, contradicting $\left(P_{6}\right)$, property satisfied by any quantifier.

Definition 5.7 Let $\mathcal{D}_{M}=(\mathcal{B}, \mathcal{R},\langle\rangle, \exists)$ be a monadic dynamic algebra. Then $\mathcal{D}_{M}^{1}=\left(\mathcal{B}^{1}, \mathcal{R}^{1},\langle\rangle, \exists^{1}\right)$, with

$$
\exists^{1}=\left(\exists_{0},\left.\exists_{1}\right|_{\mathcal{B}^{1}},\left.\exists_{2}\right|_{\mathcal{R}^{1}}\right),
$$

is a monadic dynamic subalgebra of $\mathcal{D}_{M}$ if $\mathcal{D}^{1}$ is a dynamic subalgebra of $\mathcal{D}$ and $\exists_{0} \in R^{1}$.

Lemma 5.8 If $\mathcal{D}_{M}=(\mathcal{B}, \mathcal{R},\langle\rangle, \exists)$ is a separable monadic dynamic algebra, where $\exists_{2}(R)$ is closed under the operation ${ }^{*}$, the range $\exists(\mathcal{D})=\left(\exists_{1}(\mathcal{B}), \exists_{2}(\mathcal{R}),\langle\rangle, \exists\right)$ is a monadic dynamic subalgebra of $\mathcal{D}_{M}$.

Pro of. By property $\left(P_{7}\right), \exists_{1}(\mathcal{B})$ is a Boolean subalgebra of $\mathcal{B}$.

We have to prove that:

(i) If $p \in \exists_{1}(B)$ and $a \in \exists_{2}(R)$, then $a p \in \exists_{1}(B)$.

(ii) If $a, b \in \exists_{2}(R)$, then $a \cup b, a b \in \exists_{2}(R)$.

(iii) $\exists_{1} \in \exists_{2}(R)$.

The proof of conditions (i) and (ii) is straightforward.

Condition (iii) is satisfied, since condition $\left(\exists_{2}\left(\exists_{1}\right)\right) p=\exists_{1} p$, for every $p \in B$ implies $\exists_{1}=\exists_{2}\left(\exists_{1}\right)$ in the separable monadic dynamic algebra $\mathcal{D}_{M}$.

In the last lemma the condition on * is essential, as the next example shows. 
Example 5.9 Let $\mathcal{D}_{M}=\left(S, \mathcal{P}(S), \mathcal{P}(S \times S),\langle\rangle, \exists_{s}\right)$ be the full monadic Kripke structure, where $S=\{1,2\}$, $\exists_{s}=\left(\exists_{s 1}, \exists_{s 2}\right)$ and $\exists_{s 1}$ is the simple Boolean quantifier. Since $\exists_{s 2}(\mathcal{P}(S \times S))$ is not closed under the operation ${ }^{*}, \exists_{s 2}(\mathcal{D})$ is not a dynamic subalgebra of $\mathcal{D}$. In fact, let $a=\{(1,2),(2,2)\}$. We have $a=\exists_{s 2}(a)$ and so $a \in \exists_{s 2}(P(S \times S))$. But, $a^{*} p=\min \{q: p \vee a q \leq q\}$ and so $a^{*}\{1\}=\{1\}$. Since

$$
\left(\exists_{s 2}\left(a^{*}\right)\right)\{1\}=\exists_{s 1}\{1\}=\{1,2\},
$$

we may conclude that $\exists_{s 2}\left(a^{*}\right) \neq a^{*}$. This yields $a^{*} \notin \exists_{s 2}(P(S \times S))$ by Proposition 5.5, 7 .

The notion of a homomorphism between monadic dynamic algebras is described as expected.

Definition 5.10 Let $\mathcal{D}_{M}=(\mathcal{B}, \mathcal{R},\langle\rangle, \exists)$ and $\mathcal{D}_{M}^{\prime}=\left(\mathcal{B}^{\prime}, \mathcal{R}^{\prime},\langle\rangle^{\prime}, \exists^{\prime}\right)$ be monadic dynamic algebras and let $h_{1}: B \longrightarrow B^{\prime}$ and $h_{2}: R \longrightarrow R^{\prime}$ be functions. The pair $h=\left(h_{1}, h_{2}\right)$ is a homomorphism between the monadic dynamic algebras $\mathcal{D}_{M}$ and $\mathcal{D}_{M}^{\prime}$ (monadic dynamic homomorphism) if it satisfies the following conditions:

1. $h$ is a dynamic homomorphism between $\mathcal{D}$ and $\mathcal{D}^{\prime}$.

2. $h_{2}\left(\exists_{0}\right)=\exists_{0}^{\prime}$.

Proposition 5.11 Each homomorphism $h=\left(h_{1}, h_{2}\right)$ between the monadic dynamic algebras $\mathcal{D}_{M}$ and $\mathcal{D}_{M}^{\prime}$ satisfies

$$
h_{1}\left(\exists_{1}(p)\right)=\exists_{1}^{\prime}\left(h_{1}(p)\right), \quad \text { for every } p \in B
$$

and

$$
h_{2}\left(\exists_{2}(a)\right)=\exists_{2}^{\prime}\left(h_{2}(a)\right), \quad \text { for every } a \in R .
$$

Definition 5.12 Let $\mathcal{D}_{M}=(\mathcal{B}, \mathcal{R},\langle\rangle, \exists)$ be a monadic dynamic algebra. A congruence $\theta=\left(\theta_{1}, \theta_{2}\right)$ on $\mathcal{D}_{M}$ (monadic dynamic congruence) is a (dynamic) congruence on $\mathcal{D}$.

Remark 5.13 It is obvious that, for any congruence $\theta$, in any monadic dynamic algebra $\mathcal{D}_{M}=(\mathcal{B}, \mathcal{R},\langle\rangle, \exists)$ we have:

(i) Whenever $p \theta_{1} q$, then $\exists_{1}(p) \theta_{1} \exists_{1}(q)$, for $p, q \in B$.

(ii) Whenever $a \theta_{2} b$, then $\exists_{2}(a) \theta_{2} \exists_{2}(b)$, for $a, b \in R$.

The homomorphism theorem and the consequent definition of monadic quotient algebra is stated and proved as usual. If $\mathcal{D}_{M}=(\mathcal{B}, \mathcal{R},\langle\rangle, \exists)$ is a monadic dynamic algebra and $\theta=\left(\theta_{1}, \theta_{2}\right)$ is a (monadic dynamic) congruence on $\mathcal{D}_{M}$, we form the quotient algebra

$$
\mathcal{Q}_{M}=\left(\mathcal{B} / \theta_{1}, \mathcal{R} / \theta_{2},\langle\rangle, \exists^{\prime}\right)
$$

where $\mathcal{B} / \theta_{1}=\left\{[p]_{\theta_{1}}: p \in B\right\}, \mathcal{R} / \theta_{2}=\left\{[a]_{\theta_{2}}: a \in R\right\}, \exists_{0}^{\prime}:=\left[\exists_{0}\right]_{\theta_{2}}, \exists_{1}^{\prime}$ is an operator of $\mathcal{B} / \theta_{1}$ into $\mathcal{B} / \theta_{1}$ defined by $\exists_{1}^{\prime}\left([p]_{\theta_{1}}\right):=\left[\exists_{1} p\right]_{\theta_{1}}$ and $\exists_{2}^{\prime}$ is an operator of $\mathcal{R} / \theta_{2}$ into $\mathcal{R} / \theta_{2}$ defined by $\exists_{2}^{\prime}\left([a]_{\theta_{2}}\right):=\left[\exists_{2}(a)\right]_{\theta_{2}}$. The remaining operations for quotient algebras are defined as usual.

To ascertain that $\mathcal{Q}_{M}$ is a monadic quotient dynamic algebra we only have to confirm that $\exists^{\prime}$ is a quantifier on $\mathcal{Q}_{M}$. That follows immediately by the fact that $\exists^{\prime}$ is the image under a homomorphism of the quantifier $\exists$ of $\mathcal{D}_{M}$.

In a dynamic algebra $\mathcal{D}=(\mathcal{B}, \mathcal{R},\langle\rangle)$ we define the congruences $\Delta_{B}$ and $\nabla_{B}$ on $B$ and $\Delta_{R}$ and $\nabla_{R}$ on $R$ as expected:

$$
\begin{aligned}
& \Delta_{B}=\{(p, p): p \in B\}, \quad \nabla_{B}=\{(p, q): p, q \in B\}, \\
& \Delta_{R}=\{(a, a): a \in R\}, \quad \nabla_{R}=\{(a, b): a, b \in R\} .
\end{aligned}
$$

One can easily show that the pairs $\left(\Delta_{B}, \Delta_{R}\right),\left(\nabla_{B}, \nabla_{R}\right)$ and $\left(\nabla_{B}, \Delta_{R}\right)$ are congruences on $\mathcal{D}$, but, usually, $\left(\Delta_{B}, \nabla_{R}\right)$ is not a congruence on $\mathcal{D}$.

Remark 5.14 We consider the dynamic algebra $(\{0\},\{0\},\langle\rangle)$ to be the degenerated dynamic algebra.

Definition 5.15 A non-degenerated dynamic algebra is simple if it has exactly two congruences.

Theorem 5.16 The algebras $(\mathcal{B},\{a\},\langle\rangle)$ with $\mathcal{B}$ arbitrary and $a=\exists_{s 1}$ (the simple Boolean quantifier) are the unique simple separable dynamic algebras. 
Pro of. Let $\mathcal{D}=(\mathcal{B}, \mathcal{R},\langle\rangle)$ be a separable dynamic algebra

(i) If $B=\{0\}$, then $R=\{0\}$, and so $\mathcal{D}$ is the degenerated dynamic algebra (it admits only one congruence).

(ii) If $B=\{0,1\}$, then $R=\left\{\operatorname{id}_{R}\left(=\exists_{s 1}\right)\right\}$ or $R=\left\{0, \operatorname{id}_{R}\right\}$.

In the case $R=\left\{\operatorname{id}_{R}\right\},\left(\Delta_{B}, \Delta_{R}\right)$ and $\left(\nabla_{B}, \nabla_{R}\right)$ are the unique congruences on $\mathcal{D}$, and therefore, $\mathcal{D}$ is simple.

In the case $R=\left\{0, \operatorname{id}_{R}\right\}$, since $\Delta_{R} \neq \nabla_{R},\left(\Delta_{B}, \Delta_{R}\right),\left(\nabla_{B}, \nabla_{R}\right)$ and $\left(\nabla_{B}, \Delta_{R}\right)$ are different congruences on $\mathcal{D}, \mathcal{D}$ is not simple.

(iii) For $B \notin\{\{0\},\{0,1\}\}$ we have:

1. If the cardinality of the set $R$ is greater than 1 , then $R$ admits $\nabla_{R}$ and $\Delta_{R}$ as distinct congruences. Immediately $\left(\Delta_{B}, \Delta_{R}\right),\left(\nabla_{B}, \nabla_{R}\right)$ and $\left(\nabla_{B}, \Delta_{R}\right)$ are different congruences on $\mathcal{D}$ and, therefore, $\mathcal{D}$ is not a simple dynamic algebra.

2. In the case $R=\{a\}$ we have only one congruence $\Delta_{R}\left(=\nabla_{R}\right)$ on $\mathcal{R}$.

(a) Let $a=\exists_{s 1}$ and $\theta_{B}$ be a congruence on $\mathcal{B}$ with $\theta_{B} \neq \Delta_{B}$ and $\theta_{B} \neq \nabla_{B}$. Since $\theta_{B} \neq \nabla_{B}$ we have $[0]_{\theta_{B}} \neq[1]_{\theta_{B}}$ and, from $\theta_{B} \neq \Delta_{B}$, we know that there exists $p \neq 0$ such that $0 \theta_{B} p$. Since $a 0=0$ and $a p=1$ are in different congruence classes, $\left(\theta_{B}, \Delta_{R}\right)$ is not a dynamic congruence. Therefore, $\mathcal{D}$ is a simple dynamic algebra.

(b) Now, let $a \neq \exists_{s 1}$. Since $a=a \cup a=a ; a=a^{*}$ and $a^{*} p \geq p$, we have $a p \geq p$, for every $p \in B$. Therefore, there exists an element $p \notin\{0,1\}$ with $a p=p^{\prime}$ and $p \leq p^{\prime}<1$. But

$$
a p^{\prime}=a(a p)=(a a) p=a p=p^{\prime} .
$$

Let $I_{p^{\prime}}$ be the principal Boolean ideal generated by $p^{\prime}$ in $\mathcal{B}$, i. e.

$$
I_{p^{\prime}}=\left\{q \in B: q \leq p^{\prime}\right\} .
$$

For every $i \in I_{p^{\prime}}$ we have $i \leq p^{\prime}$, and so $a i \leq a p^{\prime}=p^{\prime}$, that is $a i \in I_{p^{\prime}}$. Let $\theta_{p^{\prime}}$ denote the Boolean congruence defined by $s \theta_{p^{\prime}} t$ if and only if $s \vee i=t \vee i$, for some $i \in I_{p^{\prime}}$. From $s \theta_{p^{\prime}} t$ we get $a s \vee a i=a t \vee a i$ with ai $\in I_{p^{\prime}}$, yielding as $\theta_{p^{\prime}}$ at. Therefore, $\left(\theta_{p^{\prime}}, \Delta_{R}\right)$ is a congruence on $\mathcal{D}$ different from $\left(\Delta_{B}, \Delta_{R}\right)$ and $\left(\nabla_{B}, \Delta_{R}\right)$ and so $\mathcal{D}$ is not simple.

Corollary 5.17 The unique simple separable monadic dynamic algebras are $\left(\mathcal{B},\left\{\exists_{s 1}\right\},\langle\rangle, \exists_{s}\right)$, for arbitrary Boolean algebras $\mathcal{B}, \exists_{s}=\left(\exists_{s 1}, \exists_{s 2}\right)$ and $\exists_{s 1}$ the simple Boolean quantifier.

\section{Duality}

From now on we will assume that all dynamic algebras are endowed with a reversion operator.

\subsection{Stone space of a separable dynamic algebra with reversion}

By Stone's Representation Theorem, there exists a bijective correspondence between Boolean algebras $\mathcal{B}$ and Boolean spaces $\mathcal{X}$ in such a way that $\mathcal{B}$ is isomorphic to the algebra of all the clopen subsets of the corresponding space $\mathcal{X}$. The precise statement of the facts is as follows: If $S$ is the set of all the ultrafilters of $B$, Stone's Representation Theorem endows $S$ with a topology $\mathcal{T}$ such that the Boolean algebra $\mathcal{B}$ becomes isomorphic to the Boolean algebra $\mathcal{S}$ of all clopen subsets of $\mathcal{X}=(S, \mathcal{T})$ by means of the mapping

$$
h: \mathcal{B} \longrightarrow \mathcal{S}, \quad p \longmapsto h(p)=p^{\prime}=\{F \in S: p \in F\} .
$$

There exists a natural isomorphism between the dual algebra $\mathcal{S}$ of a Boolean space $\mathcal{X}$ and the set of all continuous functions from $\mathcal{X}$ into $2=\{0,1\}$. In order to interpret this assertion we have to endow 2 with a topology. The topology introduced in 2 is the discrete one, i. e. all the four subsets of 2 are open (therefore closed). Thus, 2 becomes a (discrete) Boolean space. The mentioned isomorphism maps each clopen subset of $\mathcal{X}$ to its characteristic function. 
Therefore, if $\mathcal{B}$ is a Boolean algebra, $S$ the set of the ultrafilters of $B$ and $\mathcal{S}$ the algebra of the clopen subsets of $(S, \mathcal{T})$ (where $\mathcal{T}$ is the topology induced by Stone's Representation Theorem), we have the isomorphisms

$$
\begin{aligned}
& \mathcal{B} \longrightarrow \mathcal{S} \longrightarrow 2^{S}, \quad p \longmapsto\{F \in S: p \in F\} \longmapsto f_{p}: S \longrightarrow 2, \\
& G \longmapsto f_{p}(G)= \begin{cases}1 & \text { if } p \in G, \\
0 & \text { if } p \notin G .\end{cases}
\end{aligned}
$$

In the following, we are going to identify each Boolean algebra $\mathcal{B}$ with the algebra of all the continuous functions of its dual space into 2 .

Our next aim is to determine the entity corresponding to a quantifier $\exists$ on the dual space of a given monadic Boolean algebra $\mathcal{B}$. We begin by recalling the notion of hemimorphism. A Boolean hemimorphism is a mapping from a Boolean algebra $\mathcal{B}$ into a Boolean algebra $\mathcal{C}$ such that $f$ is normal $(f 0=0)$ and additive $(f(p \vee q)=f p \vee f q$, for every $p, q$ in $B)$. Obviously, every quantifier is a hemimorphism from a Boolean algebra $\mathcal{B}$ into itself. A Boolean relation is a relation $\varphi$ from a Boolean space $\mathcal{Y}$ to a Boolean space $\mathcal{X}$ such that the direct image of each point of $\mathcal{Y}$ is a closed set in $\mathcal{X}$ and the inverse image of each clopen set in $\mathcal{X}$ is a clopen set in $\mathcal{Y}$.

Let $\mathcal{B}$ and $\mathcal{C}$ be Boolean algebras having, respectively, $\mathcal{X}=\left(S_{B}, \mathcal{T}_{B}\right)$ and $\mathcal{Y}=\left(S_{C}, \mathcal{T}_{C}\right)$ as dual spaces. If $f$ is a hemimorphism from $\mathcal{B}$ into $\mathcal{C}$, then its dual, denoted by $f^{\delta}$, is the relation from $\mathcal{Y}$ in $\mathcal{X}$ defined by

$$
f^{\delta}=\bigcap_{p \in B}\left\{(F, G) \in S_{C} \times S_{B}: p(G) \leq f p(F)\right\},
$$

i. e. $F f^{\delta} G$ if and only if $p(G) \leq f p(F)$, for every $p \in B$. It can be proved that $f^{\delta}$ is a Boolean relation from $\mathcal{Y}$ in $\mathcal{X}$, see [1].

Every quantifier is a hemimorphism. Moreover, Halmos [1] proved that the dual of a quantifier $\exists$ on $\mathcal{B}$ is the equivalence relation on $S_{B}$

$$
\exists^{\delta}=\bigcap_{p \in B}\left\{\left(F_{1}, F_{2}\right) \in S_{B} \times S_{B}: \exists p\left(F_{2}\right)=\exists p\left(F_{1}\right)\right\} .
$$

We recall the following notions, cf. [7].

Definition 6.1 A topological Kripke structure is a Kripke structure $(S, \mathcal{B}, \mathcal{R})$ endowed with the topology $\mathcal{S}$ in $S$ generated by $\mathcal{B}$.

Definition 6.2 A dynamic space is a topological Kripke structure $\mathcal{A}=(S, \mathcal{B}, \mathcal{R})$ such that

1. $(S, \mathcal{S})$ is Hausdorff and compact;

2. every element of $\mathcal{R}$ is closed in the product topology $\mathcal{S} \times \mathcal{S}$.

Let $\mathcal{D}_{M}=(\mathcal{B}, \mathcal{R},\langle\rangle, \exists)$ be a separable monadic dynamic algebra and $S$ the set of all the ultrafilters of $\mathcal{B}$. Each $a \in R$ is a hemimorphism in $\mathcal{B}$ satisfying certain additional properties, and its corresponding entity in the dual dynamic space is

$$
\begin{aligned}
a^{\delta} & =\bigcap_{p \in B}\{(F, G) \in S \times S: p(G) \leq a p(F)\} \\
& =\{(F, G) \in S \times S: p(G) \leq a p(F), \text { for every } p \in B\} .
\end{aligned}
$$

Since $p(G) \leq a p(F)$ is false only if $p(G)=1$ and $a p(F)=0$, the condition

$$
p(G) \leq a p(F), \quad \text { for every } p \in B
$$

is equivalent to

$$
p(G)=1 \Rightarrow a p(F)=1, \quad \text { for every } p \in B,
$$

i.e.

$$
p \in G \Rightarrow a p \in F, \quad \text { for every } p \in B .
$$

Therefore,

$$
a^{\delta}=\{(F, G): p \in G \Rightarrow a p \in F\} .
$$


Similarly, we conclude that the dual of $\exists_{1}$ is

$$
\begin{aligned}
\exists_{1}^{\delta} & =\bigcap_{p \in B}\left\{(F, G) \in S \times S: \exists_{1} p(G)=\exists_{1} p(F)\right\} \\
& =\left\{(F, G): \exists_{1} p \in F \text { if and only if } \exists_{1} p \in G, \text { for every } p \in B\right\} .
\end{aligned}
$$

Now we are led to extend the isomorphism defined by Kozen [6] between the class of the separable monadic dynamic algebras and the class of the (possibly non-standard) monadic Kripke structures by letting

1. $p^{\prime}=\{F \in S: p \in F\}$, for $p \in B$;

2. $a^{\prime}=\{(F, G) \in S \times S: p \in G \Rightarrow a p \in F\}$, for $a \in R$;

3. $B^{\prime}=\left\{p^{\prime}: p \in B\right\}$;

4. $R^{\prime}=\left\{a^{\prime}: a \in R\right\}$

5. $\exists_{1}^{\prime}=\left\{(F, G) \in S \times S: \exists_{1} p \in F\right.$ if and only if $\exists_{1} p \in G$, for every $\left.p \in B\right\}$;

6. $\exists_{2}^{\prime}(a)=\exists_{1}^{\prime} ; a^{\prime}$

The structure $S(\mathcal{D})=\left(S, \mathcal{B}^{\prime}, \mathcal{R}^{\prime}\right)$ is a dynamic space called the Stone space of $\mathcal{D}$ (the dual space of $\left.\mathcal{D}\right)$, see [7], and $\mathcal{D}$ is isomorphic to the characteristic algebra of $S(\mathcal{D}), C(S(\mathcal{D}))=\left(\mathcal{B}^{\prime}, \mathcal{R}^{\prime},\langle\rangle\right)$ (cf. [6, 7, 9]). Since $\exists_{1}^{\prime}$ is the image under the homomorphism ' of a quantifier $\exists_{1}$, then $\exists_{1}^{\prime}$ is a quantifier on $\mathcal{B}^{\prime}$. By the definition of $\exists_{2}^{\prime}$ and by the fact that $\exists_{1}^{\prime} \in R^{\prime}$ (since $\exists_{1} \in R$ ) we conclude that $\exists^{\prime}$ is a quantifier on $C\left(S(\mathcal{D})\right.$ ) and therefore $\mathcal{D}_{M}$ is isomorphic to $C\left(S\left(\mathcal{D}_{M}\right)\right)$.

We have just asserted, for separable monadic dynamic algebras (with or without reversion):

Theorem 6.3 Every separable monadic dynamic algebra is isomorphic to a (possibly) non-standard monadic Kripke structure.

\subsection{Dynamic hemimorphisms and dynamic relations}

We have previously referred the isomorphism between the class of separable dynamic algebras $\mathcal{D}=(\mathcal{B}, \mathcal{R},\langle\rangle)$ and that of Kripke structures, identifying each Boolean element $p$ with the set $p^{\prime}=\{U \in S: p \in U\}$ and each regular element $a$ with the set $a^{\prime}=\{(U, V) \in S \times S: p \in V \Rightarrow a p \in U\}$, where $S$ represents the set of all the ultrafilters of $\mathcal{B}$. Moreover, Kozen [7] proved that if $\mathcal{A}=(S, \mathcal{B}, \mathcal{R})$ is a dynamic space, then the corresponding characteristic algebra, $C(\mathcal{A})=(\mathcal{B}, \mathcal{R},\langle\rangle)$ is a separable dynamic algebra and $S(C(\mathcal{A}))$ is homeomorphic to $\mathcal{A}$.

Now we are going to introduce the notions of hemimorphism in dynamic algebras, of dynamic relation in dynamic spaces and verify that these two entities are dually related in the class of the separable dynamic algebras.

Definition 6.4 A dynamic hemimorphism is a pair of functions $f=\left(f_{1}, f_{2}\right)$ mapping of a dynamic algebra $\mathcal{D}=(\mathcal{B}, \mathcal{R},\langle\rangle)$ into a dynamic algebra $\mathcal{D}_{1}=\left(\mathcal{B}_{1}, \mathcal{R}_{1},\langle\rangle\right)$ such that

(i) $f_{1}$ is a Boolean hemimorphism;

(ii) $f_{2} 0=0$ whenever $0 \in R$;

(iii) $f_{2}(a \cup b)=f_{2} a \cup f_{2} b$, for every $a, b \in R$.

Definition 6.5 If $\mathcal{D}=(\mathcal{B}, \mathcal{R},\langle\rangle)$ is a dynamic algebra and $S$ is the set of all the ultrafilters of $\mathcal{B}$, we say that every set of the form

$$
a^{\prime}=\{(U, V) \in S \times S: p \in V \Rightarrow a p \in U\}
$$

for some $a \in R$ is a closed action of the dual space of $\mathcal{D}$.

Definition 6.6 A dynamic relation between dynamic spaces $\left(S_{0}, \mathcal{B}_{0}, \mathcal{R}_{0}\right)$ and $(S, \mathcal{B}, \mathcal{R})$ is a pair $\varphi=\left(\varphi_{1}, \varphi_{2}\right)$ such that

1. $\varphi_{1}$ is a Boolean relation of $S_{0}$ in $S$;

2. $\varphi_{2}$ is a relation of $S_{0} \times S_{0}$ in $S \times S$ such that

(a) the inverse image under $\varphi_{2}$ of a closed action of $S \times S$ is a closed action of $S_{0} \times S_{0}$;

(b) for each $(U, V) \notin \varphi_{2}\left(U_{0}, V_{0}\right)$, there exists $b \in R$ such that $(U, V) \in b^{\prime}$ and $b^{\prime} \cap \varphi_{2}\left(U_{0}, V_{0}\right)=\emptyset$, for every $\left(U_{0}, V_{0}\right)$. 
Let $\mathcal{D}=(\mathcal{B}, \mathcal{R},\langle\rangle)$ and $\mathcal{D}_{0}=\left(\mathcal{B}_{0}, \mathcal{R}_{0},\langle\rangle\right)$ be separable dynamic algebras and

$$
S(\mathcal{D})=\left(S, \mathcal{B}^{\prime}, \mathcal{R}^{\prime}\right), \quad S\left(\mathcal{D}_{0}\right)=\left(S_{0}, \mathcal{B}_{0}^{\prime}, \mathcal{R}_{0}^{\prime}\right)
$$

their corresponding Stone spaces.

If $f=\left(f_{1}, f_{2}\right)$ is a hemimorphism of $\mathcal{D}$ into $\mathcal{D}_{0}$, its dual, denoted by $f^{\delta}$, is the pair $\left(f_{1}^{\delta}, f_{2}^{\delta}\right)$, where $f_{1}^{\delta}$ is the relation of $S_{0}$ in $S$ defined by

$$
f_{1}^{\delta}=\left\{\left(U_{0}, U\right) \in S_{0} \times S: p \in U \Rightarrow f_{1} p \in U_{0}, \text { for every } p \in B\right\}
$$

and $f_{2}^{\delta}$ is the relation of $S_{0} \times S_{0}$ in $S \times S$ defined by

$$
f_{2}^{\delta}=\left\{\left(\left(U_{0}, V_{0}\right),(U, V)\right) \in\left(S_{0} \times S_{0}\right) \times(S \times S):(U, V) \in a^{\prime} \Rightarrow\left(U_{0}, V_{0}\right) \in\left(f_{2} a\right)^{\prime}, a \in R\right\} .
$$

If $\varphi=\left(\varphi_{1}, \varphi_{2}\right)$ is a relation of the dynamic space $S\left(\mathcal{D}_{0}\right)$ into the dynamic space $S(D)$, its dual, denoted by $\varphi^{\delta}$, is the pair $\left(\varphi_{1}^{\delta}, \varphi_{2}^{\delta}\right)$, where $\varphi_{1}^{\delta}$ is the map from $B$ in $B_{0}$ given by

$$
\left(\varphi_{1}^{\delta} p\right)^{\prime}=\varphi_{1}^{-1} p^{\prime}
$$

and $\varphi_{2}^{\delta}$ is the map from $R$ into $R_{0}$ given by

$$
\left(\varphi_{2}^{\delta} a\right)^{\prime}=\varphi_{2}^{-1} a^{\prime}
$$

The next theorem is a fundamental result in the dynamic duality theory.

Theorem 6.7 If $f$ is a dynamic hemimorphism between the separable dynamic algebras $\mathcal{D}=(\mathcal{B}, \mathcal{R},\langle\rangle)$ and $\mathcal{D}_{0}=\left(\mathcal{B}_{0}, \mathcal{R}_{0},\langle\rangle\right)$ with $R$ and $R_{0}$ finite, then $f^{\delta}$ is a dynamic relation, and $\left(f^{\delta}\right)^{\delta}=f$. If $\varphi$ is a dynamic relation, then $\varphi^{\delta}$ is a dynamic hemimorphism and $\left(\varphi^{\delta}\right)^{\delta}=\varphi$. If $f$ and $\varphi$ are mutually dual, then

(*) $\quad \varphi_{1}^{-1} p^{\prime}=\left(f_{1} p\right)^{\prime}$

and $\varphi_{2}^{-1} a^{\prime}=\left(f_{2} a\right)^{\prime}$ for every $p \in B$ and $a \in R$.

Proof. We follow [1] in the proof for the Boolean part (using the dual space $\mathcal{S}$ instead of $2^{S}$ ).

(i) We begin by showing that, if $f$ is a dynamic hemimorphism, then $f^{\delta}$ is a dynamic relation. Let $f$ be a dynamic hemimorphism of $\mathcal{D}=(\mathcal{B}, \mathcal{R},\langle\rangle)$ into $\mathcal{D}_{0}=\left(\mathcal{B}_{0}, \mathcal{R}_{0},\langle\rangle\right)$. Then

$$
\begin{aligned}
f_{1}^{\delta} & =\left\{\left(U_{0}, U\right) \in S_{0} \times S: p \in U \Rightarrow f_{1} p \in U_{0}, \text { for every } p \in B\right\} \\
& =\bigcap_{p \in B}\left\{\left(U_{0}, U\right) \in S_{0} \times S: p \in U \Rightarrow f_{1} p \in U_{0}\right\} .
\end{aligned}
$$

Let $U_{0} \in S_{0}\left(U_{0}\right.$ is a point in $\left.S_{0}\right)$. Since

$$
f_{1}^{\delta} U_{0}=\bigcap_{p \in B}\left\{U \in S: p \in U \Rightarrow f_{1} p \in U_{0}\right\},
$$

to prove that this set is closed it is sufficient to prove that each one of the sets $\left\{U \in S: p \in U \Rightarrow f_{1} p \in U_{0}\right\}$, denoted by $F_{p}$, is closed. In fact, if $f_{1} p \in U_{0}$, then

$$
p \in U \Rightarrow f_{1} p \in U_{0}
$$

is true for every $U \in S$, therefore $F_{p}=S$, a closed set. If $f_{1} p \notin U_{0}$, then

$$
p \in U \Rightarrow f_{1} p \in U_{0}
$$

is only true if we have $p \notin U$. Consequently,

$$
F_{p}=\{U \in S: p \notin U\}=\{U \in S: p \in U\}^{\mathrm{c}}=p^{\mathrm{c}}
$$

which is closed (since $p^{\prime}$ is a clopen set). 
We have confirmed that the image under $f_{1}^{\delta}$ of a point is closed. Now we will verify that the inverse image under $f_{1}^{\delta}$ of a clopen set is a clopen set. Let $P$ be a clopen subset of $S$. Then $P=p^{\prime}$ for some $p \in B$. We must prove that $\left(f_{1}^{\delta}\right)^{-1} p^{\prime}$ is a clopen set of $S_{0}$. If we establish that $(*)$ is valid for $f_{1}^{\delta}$ in the role of $\varphi_{1}$, i.e.

$$
\left(f_{1}^{\delta}\right)^{-1} p^{\prime}=\left(f_{1} p\right)^{\prime},
$$

we have the required, since $\left(f_{1} p\right)^{\prime}$ is a clopen set. If $p=0$, then $p^{\prime}=\emptyset$, and therefore, $\left(f_{1}^{\delta}\right)^{-1} 0^{\prime}=\emptyset$. Since $f_{1}$ is normal, $f_{1} 0=0$ and so, $\left(f_{1} 0\right)^{\prime}=\emptyset$. Then $\left(f_{1}^{\delta}\right)^{-1} 0^{\prime}=\left(f_{1} 0\right)^{\prime}$. If $p \neq 0$, then $p^{\prime} \neq \emptyset$ since there always exists an ultrafilter containing the element $p$ (the ultrafilter containing the filter generated by $p$ ). If $U_{0} \in\left(f_{1}^{\delta}\right)^{-1} p^{\prime}$, then there exists $U \in p^{\prime}$ such that $\left(U_{0}, U\right) \in f_{1}^{\delta}$, i. e. $q \in U$ implies $f_{1} q \in U_{0}$, for every $q \in B$. Since $p \in U$, then $f_{1} p \in U_{0}$. Therefore $U_{0} \in\left(f_{1} p\right)^{\prime}$. We have just proved that $\left(f_{1}^{\delta}\right)^{-1} p^{\prime} \subseteq\left(f_{1} p\right)^{\prime}$, for every $p \neq 0$ in $B$. To prove that $\left(f_{1} p\right)^{\prime} \subseteq\left(f_{1}^{\delta}\right)^{-1} p^{\prime}$ we are going to show that, if an element does not belong to $\left(f_{1}^{\delta}\right)^{-1} p^{\prime}$, then it does not belong to $\left(f_{1} p\right)^{\prime}$, also. Equivalently,

$$
\text { if } U_{0} \notin\left(f_{1}^{\delta}\right)^{-1} p^{\prime} \text {, then } f_{1} p \notin U_{0} \text {. }
$$

If $U_{0} \notin\left(f_{1}^{\delta}\right)^{-1} p^{\prime}$, then $\left(U_{0}, U\right) \notin f_{1}^{\delta}$, for every $U \in p^{\prime}$. Then, for every $U \in p^{\prime}$, there exists $p_{U}$ such that $p_{U} \in U$ and $f_{1} p_{U} \notin U_{0}$. Thus

$$
p^{\prime} \subseteq \bigcup_{U \in p^{\prime}}\left\{Z \in S: p_{U} \in Z\right\}=\bigcup_{U \in p^{\prime}} p_{U}^{\prime} .
$$

This is an open cover of $p^{\prime}$. Since $p^{\prime}$ is compact (because it is closed) there exists a finite subset $\left\{U_{1}, U_{2}, \ldots, U_{n}\right\}$ of $p^{\prime}$ such that

$$
p^{\prime} \subseteq \bigcup_{j=1}^{n}\left\{Z \in S: p_{U_{j}} \in Z\right\}=\bigcup_{j=1}^{n} p_{U_{j}}^{\prime}
$$

By hypothesis $p^{\prime}$ is not empty and so $n \neq 0$, i. e. the finite set is not empty. Let

$$
\tilde{p}=\bigvee_{j=1}^{n} p_{U_{j}} .
$$

Therefore, we have

$$
p \leq \tilde{p}
$$

and since $f_{1}$ is monotone,

$$
f_{1} p \leq f_{1} \tilde{p} .
$$

Then, for every $U_{0} \in S_{0}$,

$$
f_{1} p \in U_{0} \Rightarrow f_{1} \tilde{p} \in U_{0} .
$$

The additivity of $f_{1}$ allows us to write

$$
f_{1} \tilde{p}=f_{1}\left(\bigvee_{j=1}^{n} p_{U_{j}}\right)=\bigvee_{j=1}^{n} f_{1} p_{U_{j}} .
$$

Since, for every $U \in p^{\prime}$, we have $f_{1} p_{U} \notin U_{0}$, then $\sim\left(f_{1} p_{U}\right) \in U_{0}$. Consequently,

$$
\bigwedge_{j=1}^{n}\left(\sim\left(f_{1} p_{U_{j}}\right)\right) \in U_{0} .
$$

Therefore, $f_{1} \tilde{p} \notin U_{0}$.

Since $f_{1} \tilde{p} \notin U_{0}$, then, by (2), we have $f_{1} p \notin U_{0}$, as required.

Now we are going to analyze $f_{2}^{\delta}$. If we prove that, for every $a \in R$,

$$
\left(f_{2}^{\delta}\right)^{-1} a^{\prime}=\left(f_{2} a\right)^{\prime},
$$

then we establish that the inverse image under $f_{2}^{\delta}$ of a closed action is a closed action. 
If $a=0$, then $a^{\prime}=\emptyset$ and therefore, $\left(f_{2}^{\delta}\right)^{-1} 0^{\prime}=\emptyset$. But since $\left(f_{2} 0\right)^{\prime}=0^{\prime}=\emptyset$ we have $\left(f_{2}^{\delta}\right)^{-1} 0^{\prime}=\left(f_{2} 0\right)^{\prime}$.

If $a \neq 0$, then $a^{\prime} \neq \emptyset$ (since ${ }^{\prime}$ is an isomorphism). We show that $\left(f_{2}^{\delta}\right)^{-1} a^{\prime} \subseteq\left(f_{2} a\right)^{\prime}$. Let $\left(U_{0}, V_{0}\right) \in\left(f_{2}^{\delta}\right)^{-1} a^{\prime}$. Then there exists $(U, V) \in a^{\prime}$ such that $\left(\left(U_{0}, V_{0}\right),(U, V)\right) \in f_{2}^{\delta}$, i.e. $(U, V) \in b^{\prime}$ implies $\left(U_{0}, V_{0}\right) \in\left(f_{2} b\right)^{\prime}$, for every $b \in R$. Since $(U, V) \in a^{\prime}$, then $\left(U_{0}, V_{0}\right) \in\left(f_{2} a\right)^{\prime}$. Now we prove that $\left(f_{2} a\right)^{\prime} \subseteq\left(f_{2}^{\delta}\right)^{-1} a^{\prime}$. Let us assume that $\left(U_{0}, V_{0}\right) \notin\left(f_{2}^{\delta}\right)^{-1} a^{\prime}$. We want to show that $\left(U_{0}, V_{0}\right) \notin\left(f_{2} a\right)^{\prime}$. Since $\left(U_{0}, V_{0}\right) \notin\left(f_{2}^{\delta}\right)^{-1} a^{\prime}$, then for every $(U, V) \in a^{\prime}$ we have $\left(\left(U_{0}, V_{0}\right),(U, V)\right) \notin f_{2}^{\delta}$, i. e. for some $b_{U V} \in R$,

$$
(U, V) \in b_{U V}^{\prime} \quad \text { and } \quad\left(U_{0}, V_{0}\right) \notin\left(f_{2} b_{U V}\right)^{\prime} .
$$

Thus

$$
a^{\prime} \subseteq \bigcup_{(U, V) \in a^{\prime}}\left\{\left(Z_{1}, Z_{2}\right): p \in Z_{2} \Rightarrow b_{U V} p \in Z_{1}\right\}=\bigcup_{(U, V) \in a^{\prime}} b_{U V}^{\prime}
$$

Let

$$
\tilde{b}=\bigcup_{(U, V) \in a^{\prime}} b_{U V}
$$

( $\tilde{b}$ is defined since $R$ is finite). Therefore, from $a^{\prime} \subseteq(\tilde{b})^{\prime}$ we get $a \leq \tilde{b}$. Since $f_{2}$ is monotone, $f_{2} a \leq f_{2} \tilde{b}$ and so,

$$
\left(f_{2} a\right)^{\prime} \subseteq\left(f_{2} \tilde{b}\right)^{\prime} .
$$

Now we prove the following: If $\left(U_{0}, V_{0}\right) \notin\left(f_{2} b_{U V}\right)^{\prime}$, for every $(U, V) \in a^{\prime}$, then $\left(U_{0}, V_{0}\right) \notin\left(f_{2} \tilde{b}\right)^{\prime}$.

By (4) we have $\left(f_{2} \tilde{b}\right)^{\prime}=\bigcup_{(U, V) \in a^{\prime}}\left(f_{2} b_{U V}\right)^{\prime}$, i. e. $\left(f_{2} \tilde{b}\right)^{\prime c}=\bigcap_{(U, V) \in a^{\prime}}\left(f_{2} b_{U V}\right)^{\prime c}$.

Using (3), for every $(U, V) \in a^{\prime}$ we have $\left(U_{0}, V_{0}\right) \notin\left(f_{2} b_{U V}\right)^{\prime}$, then $\left(U_{0}, V_{0}\right) \notin\left(f_{2} \tilde{b}\right)^{\prime}$. Using (5) we have $\left(U_{0}, V_{0}\right) \notin\left(f_{2} a\right)^{\prime}$.

We proved that $\left(f_{2} a\right)^{\prime} \subseteq\left(f_{2}^{\delta}\right)^{-1} a^{\prime}$.

Now, to finish the proof that $f^{\delta}$ is a dynamic relation, we need to show that, for every $\left(U_{0}, V_{0}\right)$, whenever $(U, V) \notin f_{2}^{\delta}\left(U_{0}, V_{0}\right)$, there exists $b \in R$ such that $(U, V) \in b^{\prime}$ and $\left(U_{0}, V_{0}\right) \notin\left(f_{2}^{\delta}\right)^{-1} b^{\prime}$. But this is a consequence of the definition of $f_{2}^{\delta}$.

(ii) Let us prove that $\left(f^{\delta}\right)^{\delta}=f$. We begin by showing that $\left(f_{1}^{\delta}\right)^{\delta}=f_{1}$. It is enough to prove that $\left(f_{1}^{\delta}\right)^{\delta} p=f_{1} p$, for every $p \in B$, or either that $\left(\left(f_{1}^{\delta}\right)^{\delta} p\right)^{\prime}=\left(f_{1} p\right)^{\prime}$, for every $p \in B$. Since $\left(\left(f_{1}^{\delta}\right)^{\delta} p\right)^{\prime}=\left(f_{1}^{\delta}\right)^{-1} p^{\prime}$ and $(*)$ is satisfied with $f_{1}^{\delta}$ in the role of $\varphi_{1}$, we have $\left(f_{1}^{\delta}\right)^{-1} p^{\prime}=\left(f_{1} p\right)^{\prime}$ and therefore, $\left(\left(f_{1}^{\delta}\right)^{\delta} p\right)^{\prime}=\left(f_{1} p\right)^{\prime}$.

Similarly we have $\left(f_{2}^{\delta}\right)^{\delta}=f_{2}$, i.e. $\left(f_{2}^{\delta}\right)^{\delta} a=f_{2} a$, for every $a \in R$.

(iii) Let us show that if $\varphi$ is a dynamic relation, then $\varphi^{\delta}$ is a dynamic hemimorphism. We begin by showing that if $\varphi_{1}$ is a Boolean relation, then $\varphi_{1}^{\delta}$ is a Boolean hemimorphism. By definition of $\varphi_{1}^{\delta}$ we have

$$
\left(\varphi_{1}^{\delta} p\right)^{\prime}=\varphi_{1}^{-1} p^{\prime}
$$

Since the inverse image under $\varphi_{1}$ of a clopen set is a clopen set, it follows that $\varphi_{1}^{\delta}$ maps $B$ into $B_{0}$. Now we prove that $\varphi_{1}^{\delta}$ is a hemimorphism. For every $p, q \in B$ we have

$$
\left(\varphi_{1}^{\delta}(p \vee q)\right)^{\prime}=\varphi_{1}^{-1}(p \vee q)^{\prime}=\varphi_{1}^{-1}\left(p^{\prime} \cup q^{\prime}\right)=\varphi_{1}^{-1} p^{\prime} \cup \varphi_{1}^{-1} q^{\prime}=\left(\varphi_{1}^{\delta} p\right)^{\prime} \cup\left(\varphi_{1}^{\delta} q\right)^{\prime}=\left(\varphi_{1}^{\delta} p \vee \varphi_{1}^{\delta} q\right)^{\prime}
$$

and therefore, $\varphi_{1}^{\delta}(p \vee q)=\varphi_{1}^{\delta} p \vee \varphi_{1}^{\delta} q$.

Since $\left(\varphi_{1}^{\delta} 0\right)^{\prime}=\varphi_{1}^{-1} 0^{\prime}=\varphi_{1}^{-1} \emptyset=\emptyset=0^{\prime}$, then $\varphi_{1}^{\delta} 0=0$.

Similarly we get $\varphi_{2}^{\delta}(a \cup b)=\varphi_{2}^{\delta} a \cup \varphi_{2}^{\delta} b$, for every $a, b \in R$ and $\varphi_{2}^{\delta} 0=0$.

(iv) We show that $\left(\varphi^{\delta}\right)^{\delta}=\varphi$. We begin by proving that $\left(\varphi_{1}^{\delta}\right)^{\delta}=\varphi_{1}$. We know that

$$
\left(\varphi_{1}^{\delta} p\right)^{\prime}=\varphi_{1}^{-1}\{U \in S: p \in U\} \quad \text { and } \quad\left(\varphi_{1}^{\delta}\right)^{\delta}=\left\{\left(U_{0}, U\right) \in S_{0} \times S: p \in U \Rightarrow \varphi_{1}^{\delta} p \in U_{0}\right\} .
$$

(a) If $\left(U_{0}, U\right) \in \varphi_{1}$ and $p \in U$, then $U_{0} \in\left(\varphi_{1}^{\delta} p\right)^{\prime}$, i. e. $\varphi_{1}^{\delta} p \in U_{0}$. Therefore $\left(U_{0}, U\right) \in\left(\varphi_{1}^{\delta}\right)^{\delta}$.

(b) Let $\left(U_{0}, U\right) \notin \varphi_{1}$ (then $\left.U \notin \varphi_{1} U_{0}\right)$. Since $\varphi_{1} U_{0}$ is closed, whenever $\left(U_{0}, Z\right) \in \varphi_{1}$ there exists $p_{0}$ such that $U \in p_{0}^{\prime}$ and $Z \notin p_{0}^{\prime}$. Therefore $U_{0} \notin \varphi_{1}^{-1} p_{0}^{\prime}=\left(\varphi_{1}^{\delta} p_{0}\right)^{\prime}$. Thus, there exists $p_{0} \in U$ such that $\varphi_{1}^{\delta} p_{0} \notin U_{0}$. Therefore $\left(U_{0}, U\right) \notin\left(\varphi_{1}^{\delta}\right)^{\delta}$. 
It remains to be proved that $\left(\varphi_{2}^{\delta}\right)^{\delta}=\varphi_{2}$. We have

$$
\left(\varphi_{2}^{\delta}\right)^{\delta}=\left\{\left(\left(U_{0}, V_{0}\right),(U, V)\right) \in\left(S_{0} \times S_{0}\right) \times(S \times S):(U, V) \in a^{\prime} \Rightarrow\left(U_{0}, V_{0}\right) \in\left(\varphi_{2}^{\delta} a\right)^{\prime}\right\} .
$$

(a) If $\left(\left(U_{0}, V_{0}\right),(U, V)\right) \in \varphi_{2}$, whenever $(U, V) \in a^{\prime}$, we have $\left(U_{0}, V_{0}\right) \in \varphi_{2}^{-1} a^{\prime}=\left(\varphi_{2}^{\delta} a\right)^{\prime}$. Therefore

$$
\left(\left(U_{0}, V_{0}\right),(U, V)\right) \in\left(\varphi_{2}^{\delta}\right)^{\delta} .
$$

(b) If $\left(\left(U_{0}, V_{0}\right),(U, V)\right) \notin \varphi_{2}$, then $(U, V) \notin \varphi_{2}\left(U_{0}, V_{0}\right)$. But so, there exists $b \in R$ such that $(U, V) \in b^{\prime}$ and $\left(U_{0}, V_{0}\right) \notin \varphi_{2}^{-1} b^{\prime}=\left(\varphi_{2}^{\delta} b\right)^{\prime}$. Therefore $\left(\left(U_{0}, V_{0}\right),(U, V)\right) \notin\left(\varphi_{2}^{\delta}\right)^{\delta}$.

\subsection{The dual of a quantifier}

In this section we will assume that $\mathcal{D}=(\mathcal{B}, \mathcal{R},\langle\rangle)$ is a separable dynamic algebra with finite regular part and that $S$ is the set of the ultrafilters of $\mathcal{B}$. Let $f=\left(f_{1}, f_{2}\right)$ be a hemimorphism of $\mathcal{D}$ into itself, with dual $\varphi=\left(\varphi_{1}, \varphi_{2}\right)$.

We begin by analyzing the Boolean part of $f$. We define the relation $\psi_{1}$ by

$$
\psi_{1}=\left\{(U, V) \in S \times S: f_{1} p \in U \text { if and only if } f_{1} p \in V \text {, for every } p \in B\right\} \text {. }
$$

Lemma 6.8 [1] Under the previous conditions, $f_{1}$ is a quantifier on $\mathcal{B}$ if and only if $\psi_{1}=\varphi_{1}$.

Lemma 6.9 [1] Under the previous conditions, $\varphi_{1}$ is an equivalence relation on $S$ if and only if $\psi_{1}=\varphi_{1}$.

Theorem 6.10 Under the previous conditions, the hemimorphism $f=\left(f_{1}, f_{2}\right)$ defines a quantifier on the dynamic algebra $\mathcal{D}=(\mathcal{B}, \mathcal{R},\langle\rangle)$ if and only if the dynamic relation $\varphi=\left(\varphi_{1}, \varphi_{2}\right)$ satisfies the following conditions:

(i) $\varphi_{1}$ is an equivalence relation on $S$.

(ii) $\varphi_{1}$ is a closed action.

(iii) $\varphi_{2}$ is given by $\varphi_{2}^{-1} a^{\prime}:=\varphi_{1} ; a^{\prime}$, for every $a \in R$.

Pro of. We begin by assuming that $f=\left(f_{1}, f_{2}\right)$ is a quantifier on $\mathcal{D}$.

We prove (i). Since $f=\left(f_{1}, f_{2}\right)$ is a quantifier on $\mathcal{D}, f_{1}$ is a quantifier on $\mathcal{B}$, and by Lemmas 6.8 and 6.9, $\varphi_{1}$ is an equivalence relation on $S$.

Now we prove (ii). We know that $f_{1} \in R$ ( $f$ is a quantifier), and so $f_{1}^{\prime}$ is a closed action. But

$$
f_{1}^{\prime}=\left\{(U, V) \in S \times S: p \in V \Rightarrow f_{1} p \in U\right\}=f_{1}^{\delta}=\varphi_{1} .
$$

Then $\varphi_{1}$ is a closed action.

It remains to prove (iii). We have $\varphi_{2}^{-1} a^{\prime}=\left(f_{2} a\right)^{\prime}=\left(f_{1} ; a\right)^{\prime}=f_{1}^{\prime} ; a^{\prime}=\varphi_{1} ; a^{\prime}$.

Now we assume that the dynamic relation $\varphi$ satisfies (i), (ii) and (iii).

We want to prove that $f$ is a quantifier on $\mathcal{D}$, i. e. that $f_{1}$ is a quantifier on $\mathcal{B}, f_{1} \in R$ and that $f_{2}(a)=f_{1} ; a$, for every $a \in R$. Since $\varphi_{1}$ is an equivalence relation on $S$, then, by Lemmas 6.8 and 6.9, we assert that $f_{1}$ is a quantifier on $\mathcal{B}$.

Let us prove that $f_{1} \in R$. Since $\varphi_{1}$ is a closed action, then there exists $b \in R$ such that $\varphi_{1}=b^{\prime}$. Since $\varphi_{1}=f_{1}^{\delta}$, it happens that $b^{\prime}=f_{1}^{\delta}$. Therefore, $b^{\prime-1} p^{\prime}=\left(f_{1}^{\delta}\right)^{-1} p^{\prime}$, for every $p \in B$. But

$$
b^{\prime-1} p^{\prime}=\left\{U \in S:(U, V) \in b^{\prime} \text { for some } V \in p^{\prime}\right\}=b^{\prime} p^{\prime}
$$

(since we are working in Kripke structures) and $\left(f_{1}^{\delta}\right)^{-1} p^{\prime}=\left(f_{1} p\right)^{\prime}$.

Therefore, $b^{\prime} p^{\prime}=\left(f_{1} p\right)^{\prime}$, i. e. $(b p)^{\prime}=\left(f_{1} p\right)^{\prime}$, for every $p \in B$. Then $b p=f_{1} p$, for every $p \in B$. Since $\mathcal{D}$ is separable, $f_{1}=b$, and so $f_{1} \in R$ (and moreover $\varphi_{1}=f_{1}^{\prime}$ ).

It remains to be proved that $f_{2}(a)=f_{1} ; a$, for every $a \in R$. Since

$$
\left(f_{2} a\right)^{\prime}=\varphi_{2}^{-1} a^{\prime}=\varphi_{1} ; a^{\prime}=f_{1}^{\prime} ; a^{\prime}=\left(f_{1} ; a\right)^{\prime},
$$

then $f_{2}(a)=f_{1} ; a$, for every $a \in R$. 


\section{References}

[1] P. Halmos, Algebraic logic I: Monadic Boolean algebras. Compositio Math. 12, 217 - 249 (1955).

[2] B. Jónsson and A. Tarski, Boolean algebras with operators. Bull. Amer. Math. Soc. 54, 79 - 80 (1948).

[3] B. Jónsson and A. Tarski, Boolean algebras with operators, part I. Amer. J. Math. 73, 891 - 939 (1951).

[4] D. Harel, First-Order Dynamic Logic. Lecture Notes in Computer Science 68 (Springer-Verlag, 1979).

[5] D. Harel, D. Kozen, and J. Tiuryn, Dynamic Logic (MIT Press, 2000).

[6] D. Kozen, A representation theorem for models of *-free PDL. Report RC7864, IBM Research Center, Yorktown Heights, New York, 1979.

[7] D. Kozen, On the duality of dynamic algebras and Kripke models. Report RC7893, IBM Research Center, Yorktown Heights, New York, 1979.

[8] R. Parikh, Propositional dynamic logic of programs: a survey. In: Proceedings Workshop on Logic of Programs, Lecture Notes in Computer Science 125, pp. 102 - 104 (Springer-Verlag, 1981).

[9] M. C. Pinto, Álgebras Dinâmicas de Boole, de Heyting e reticulares. Representações dinâmicas. Ph. D. Dissertation, University of Coimbra, Portugal, 2000.

[10] V. R. Pratt, Dynamic algebras: examples, constructions, applications. Technical Report MIT/Laboratory for Computer Science/TM-138, 1979, pp. 1 - 33, 1979. Studia Logica 50, 571 - 605 (1992).

[11] V. R. Pratt, Dynamic algebras as a well-behaved fragment of relation algebras. In: Algebraic Logic and Universal Algebra, Lecture Notes in Computer Science 425, pp. 74 - 110 (Springer-Verlag, 1990).

[12] J. Reiterman and V. Trnková, Dynamic algebras with test. J. Comp. System Sci. 35, 229 - 242 (1987).

[13] H. Sankappanavar and S. Burris, A Course in Universal Algebra (Springer-Verlag, 1981).

[14] W. Wechler, Universal Algebra for Computer Scientists (Springer-Verlag, 1992). 